\title{
ECUACIONES ESTRUCTURALES DE LA AUTOESTIMA Y EL DESEMPEÑO LABORAL DE LOS TRABAJADORES DE LAS ÁREAS DE CIENCIAS BÁSICAS E INGENIERÍA
}

\section{STRUCTURAL EQUATIONS OF SELF-ESTEEM AND JOB PERFORMANCE OF WORKERS IN BASIC SCIENCE AND ENGINEERING AREA}

iD Zoraida Judith Huamán Gutiérrez ${ }^{1,2}$

${ }^{1}$ Universidad Nacional Mayor de San Marcos, Lima, Perú.

${ }^{2}$ Universidad Nacional del Santa, Ancash, Perú.

\section{Correspondencia:}

Mag. Zoraida Judith Huamán Gutiérrez

zhuamang@unmsm.edu.pe

2018822014@uns.edu.pe

\section{RESUMEN}

El objetivo de esta investigación fue analizar el impacto de la autoestima en el desempeño laboral de los trabajadores de las áreas de Ciencias Básicas e Ingeniería mediante la aplicación del modelo de ecuaciones estructurales. Es de tipo descriptivo correlacional de diseño no experimental y transversal. Se tomó una muestra de 179 trabajadores de un total de 331 trabajadores de las áreas de Ciencias Básicas e Ingeniería. Los resultados muestran una alta asociación entre la autoestima y el desempeño laboral de los trabajadores, teniendo así un impacto directo, mediante la estimación del modelo de ecuaciones estructurales, que presentan medidas de bondad de ajuste adecuados. Se concluye, que la autoestima presenta un impacto directo en el desempeño laboral de los trabajadores de las áreas de Ciencias Básicas e Ingeniería.

Palabras clave: autoestima, desempeño laboral, modelo de ecuaciones estructurales, trabajadores, autoconcepto

\begin{abstract}
The objective of this research was to analyze the impact of self-esteem on the work performance of workers in the areas of Basic Sciences and Engineering through the application of the structural equation model. It is of a descriptive correlational type of non-experimental and cross-sectional design. A sample of 179 workers was taken from 331 workers in the areas of Basic Sciences and Engineering. The results show a high association between self-esteem and workers' job performance, thus having a direct impact. This result was obtained by estimating the structural equations model, which present adequate goodness-of-fit measures. It concluded, that self-esteem has a direct impact on the job performance of workers in the areas of Basic Sciences and Engineering.
\end{abstract}

Key words: Self-esteem, job performance, structural equation model, workers, selfconcept 


\section{INTRODUCCIÓN}

Los modelos de ecuaciones estructurales pertenecen a la familia de modelos estadísticos multivariantes que tiene como objetivo estimar el efecto y las relaciones entre múltiples variables. Estos modelos surgen a partir de la necesidad de otorgar más flexibilidad a los modelos de regresión, los cuales se caracterizan por ser menos restrictivos, debido a que incluye errores de medida tanto en las variables dependientes como en las independientes. Matemáticamente, estos modelos son más complejos de estimar que otros modelos multivariantes como los de Regresión o Análisis factorial exploratorio y, por ello, su uso fue prolongado hasta 1973 , donde se desarrollaron programas de estimación entorno a lo gráfico como el AMOS (Cequea \& Rodriguez, 2012; Samperio, 2019; Manzano, 2017). Asimismo, dicho modelo presenta una gran ventaja, la cual es proponer el tipo y dirección de las relaciones que se espera encontrar entre las diversas variables contenidas en los modelos estructurales, con el fin de estimar los parámetros que vienen especificados por las relaciones propuestas en cuanto a la autoestima y el desempeño laboral de los trabajadores de las áreas de Ciencias Básicas e Ingeniería.

Por otro lado, la autoestima se define como la capacidad de todo ser humano para desarrollar y demostrar valía, aprecio y aceptación de sí mismo, lo cual favorece a la salud emocional y las relaciones laborales que permite vivir en armonía (Brandem, 2010; Rodriguez, 2015; Aguilar, 2008). En vista de lo señalado, la autoestima es un factor que incide en el trabajo laboral; por tanto, incide en la valía de los pensamientos, sentimientos, y experiencias que se presentan en la vida diaria.

En adición, el desempeño laboral es la manera de ejercer el trabajo eficaz para alcanzar las metas comunes instituidas con anterioridad de forma eficiente con la finalidad de lograr las metas planteadas (Pernía \& Carrera, 2014). Asimismo, el desempeño del trabajador representa el uso de sus propias capacidades y destrezas constituido por todos los esfuerzos dinámicos que realiza con el objetivo de cumplir las metas comparadas con un comportamiento concurrente dirigidas al logro de metas y de resultados en correspondencia con cada uno de los objetivos propuestos, los cuales son medibles y observables a la hora de producir, hacer o realizar un trabajo específico (Blanco, 2007; Druker, 2002). Por ello, se considera que el desempeño laboral dentro de la organización se conforma por actividades perceptibles, visibles y medibles, con los fines de conseguir los objetivos y metas.

De la misma manera, la Comisión Económica para América Latina y el Caribe (2016), reflexionó que muchos de los problemas laborales tienen una incidencia devastadora en el desarrollo integral del ser humano; es decir, las situaciones laborales que se transforman en una vulnerabilidad y maltrato al trabajador se ciernen en una complejidad de factores negativos que afectan la producción y la productividad. Además de una caída del producto bruto interno del $-0.9 \%$ lo que influye en la calidad del empleo. (p. 5).

En este marco de ideas, en este presente trabajo, se ha observado que los trabajadores administrativos de la Universidad Nacional Mayor de San Marcos debido al trabajo extenuante y exigido han expresado su cansancio y su desconfianza en el sistema laboral de la universidad; es por ello, que este panorama de trabajo estaría afectando el uso de sus conocimientos, intuiciones y percepciones como herramientas para enfrentar con sosiego y serenidad las situaciones y conflictos cotidianos. Ello ha afectado el adaptarse y aceptar los nuevos retos y no sentirse intimidado ante situaciones específicas; por lo tanto, la baja autoestima no ayuda a los trabajadores a que sean capaces de transmitir a los demás colaboradores los mensajes de forma clara y en el momento adecuado lo que piensan y sienten. Por consiguiente, en la presente investigación, se tiene como objetivo determinar la influencia de la autoestima en el desempeño de los trabajadores de las áreas de Ciencias Básicas e Ingeniería.

\section{MATERIALES Y MÉTODOS}

El estudio se trata de una investigación de enfoque cuantitativo, tipo básico, diseño 
no experimental, correlacional, pues, se han considerado las variables autoestima y desempeño laboral, las cuales son sometidas a un análisis de ecuaciones estructurales (SEM). Para su ejecución se formuló un diseño del modelo de relaciones en forma de un conjunto de ecuaciones lineales.

En relación a las técnicas e Instrumentos de recolección de datos, se utilizó la técnica de encuesta, usando como instrumentos dos cuestionarios uno de 25 preguntas para medir la autoestima y otro de 32 para el desempeño laboral. Pues, cabe recalcar que, para la estimación del modelo de ecuaciones estructurales la variable autoestima está compuesto por tres dimensiones resumidos en la suma de sus ítems, mientras que la variable desempeño laboral está compuesta con seis dimensiones resumidos en las correspondientes sumas de sus ítems, obteniéndose un total de 9 factores que fueron asociados a dos variables de estudio.

\section{RESULTADOS}

En la Tabla 1, se muestra el modelo de ecuaciones estructurales de la autoestima y el desempeño laboral de los trabajadores de las áreas de Ciencias Básicas e Ingeniería

Tabla 1

Modelo de ecuaciones estructurales de la autoestima y el desempeño laboral de los trabajadores de las áreas de Ciencias Básicas e Ingeniería, 2020

\begin{tabular}{|c|c|c|c|c|c|c|c|}
\hline & \multirow{2}{*}{ Relación } & & \multicolumn{2}{|c|}{ Coeficiente de Regresión } & \multirow{2}{*}{ E.S. } & \multirow{2}{*}{ C.R. } & \multirow{2}{*}{$\mathrm{P}$} \\
\hline & & & Estimado & Estándar & & & \\
\hline $\begin{array}{c}\text { Desempeño } \\
\text { laboral }\end{array}$ & $\leftarrow$ & Autoestima & 0.780 & 0.846 & 0.066 & 11.832 & $* * *$ \\
\hline Sí mismo & $\leftarrow$ & Autoestima & 1.000 & 0.865 & & & \\
\hline Social & $\leftarrow$ & Autoestima & 0.155 & 0.539 & 0.021 & 7.539 & $* * *$ \\
\hline Hogar & $\leftarrow$ & Autoestima & 0.300 & 0.673 & 0.023 & 13.059 & $* * *$ \\
\hline $\begin{array}{l}\text { Ayuda- } \\
\text { servicio }\end{array}$ & $\leftarrow$ & Desempeño laboral & 2.043 & 0.983 & 0.028 & 73.786 & $* * *$ \\
\hline Influencia & $\leftarrow$ & Desempeño laboral & 1.344 & 0.996 & 0.021 & 63.057 & $* * *$ \\
\hline Gerenciales & $\leftarrow$ & Desempeño laboral & 1.058 & 0.980 & 0.019 & 54.894 & $* * *$ \\
\hline Cognoscitivas & $\leftarrow$ & Desempeño laboral & 1.009 & 0.988 & 0.02 & 51.73 & $* * *$ \\
\hline Logro-acción & $\leftarrow$ & Desempeño laboral & 1.000 & 0.972 & & & \\
\hline $\begin{array}{l}\text { Eficacia } \\
\text { personal }\end{array}$ & $\leftarrow$ & Desempeño laboral & 1.061 & 0.987 & 0.020 & 52.999 & $* * *$ \\
\hline e4 & $\leftarrow \rightarrow$ & e6 & 0.270 & 0.460 & 0.105 & 2.563 & 0.01 \\
\hline e5 & $\leftarrow \rightarrow$ & e6 & 1.310 & 1.424 & 0.348 & 3.769 & $* * *$ \\
\hline e2 & $\leftarrow \rightarrow$ & e3 & 0.755 & 0.382 & 0.146 & 5.167 & $* * *$ \\
\hline
\end{tabular}

E.S. (error estándar); C.R. (Coeficiente de regresión) 
En la Tabla 1, con un valor de significancia de $p=0.05$ para todas las relaciones, se observa que la autoestima presenta un impacto positivo sobre el desempeño laboral (0.846). Así mismo, la autoestima se relaciona de manera positiva con la dimensión sí mismo (0.865), con la dimensión social (0.539) y con la dimensión hogar (0.673) de la autoestima. Por otro lado, el desempeño laboral explica de manera positiva la dimensión logro-acción (0.972), de manera positiva la dimensión ayuda-servicio (0.983), a la dimensión influencia (0.996), a la dimensión gerencial (0.980), la dimensión cognoscitiva (0.988) y se asocia de manera positiva con la dimensión eficacia personal (0.987).

$\begin{aligned} & \text { Tabla } 2 \\
& \text { Indicadores de bondad de ajuste del modelo de ecuaciones estructurales de } \\
& \text { la autoestima y el desempeño laboral de los trabajadores de las áreas de } \\
& \text { Ciencias Básicas e Ingeniería, 2020 }\end{aligned}$
\begin{tabular}{lccc}
\multicolumn{1}{c}{ Indicadores } & Medida de ajuste & Valor & $\begin{array}{c}\text { Límite } \\
\text { aceptable }\end{array}$ \\
\hline Índice de ajuste normado & NFI & 0,867 & $\geq 0,90$ \\
Índice de ajuste comparativo & GFI & 0,978 & $\geq 0,90$ \\
Índice ajustado de bondad de ajuste & AGFI & 0,958 & $\geq 0,85$ \\
Índice relativo de ajuste & RFI & 0,792 & $\geq 0,90$ \\
Ráiz cuadrada del error cuadrático medio & RMR & 3,994 & $\leq 0,10$
\end{tabular}

NFI: íńndice de ajuste normado; GFI: índice de ajuste comparativo; AGFI: índice ajustado de bondad de ajuste; RFI: índice relativo de ajuste; RMR: raíz cuadrada del error cuadrático medio.

En la Tabla 2, el modelo estructural es medido a través de cinco indicadores de ajuste: En cuanto al NFI, tiene el objetivo de medir la reducción proporcional en la capacidad de ajuste una vez que se pasa del modelo nulo al planteado. El rango de alteración de este índice está entre 0 y 1 , siendo recomendables valores superiores a 0,90 (Bentler \& Bonet, 1980). De igual modo, el GFI es un índice de la variabilidad explicada por el modelo, oscilando sus valores entre 0 (pobre ajuste) y 1 (perfecto ajuste), donde valores superiores a 0,90 indican un ajuste aceptable (Jöreskog \& Sörbom, 1986). Además, AGFI es aquel índice, donde se ajusta utilizando la relación obtenida entre los grados de libertad del modelo propuesto y los grados de libertad del modelo nulo, siendo un nivel aceptable y recomendado es un valor mayor o igual a 0.85 (Beltrán, 1986). Mientras que, el RFI proporciona valores próximos y/o mayores a 0.90 a medida que el modelo va alcanzando un buen ajuste (Bollen, 1986). Finalmente, el RMR hace referencia a la cantidad de varianza no explicada por el modelo por grado de libertad, considerando un valor aceptable menor a 0.10 (Morata, 2015).

Por consiguiente, de acuerdo a los indicadores de ajuste, se puede decir que el modelo estructural estimado es aceptable, ya que cumple al menos dos de los indicadores de bondad de ajuste.

\section{Modelo estructural}

Modelo de ecuaciones estructurales de la autoestima y el desempeño laboral de los trabajadores de las áreas de Ciencias Básicas e Ingeniería, 2020

$$
\begin{gathered}
\text { DesempeñoLaboral }=0.85 \times \text { Autoestima } \mathrm{e}_{10} \\
\text { Si mismo }=0.87 \times \text { Autoestima } \mathrm{e}_{1} \\
\text { Social }=0.54 \times \text { Autoestima }+\mathrm{e}_{2} \\
\text { Hogar }=0.67 \times \text { Autoestima }+\mathrm{e}_{3} \\
\text { LogroAcción }=0.97 \times \text { DesempeñoLaboral }+\mathrm{e}_{4} \\
\text { AyudaServicio }=0.98 \times \text { DesempeñoLaboral }+\mathrm{e}_{5} \\
\text { Influencia }=1.00 \times \text { DesempeñoLaboral }+\mathrm{e}_{6} \\
\text { Gerenciales }=0.98 \times \text { DesempeñoLaboral }+\mathrm{e}_{7} \\
\text { Cognitivas }=0.99 \times \text { DesempeñoLaboral }+\mathrm{e}_{8} \\
\text { EficaciaPersonal }=0.99 \times \text { DesempeñoLaboral }+\mathrm{e}_{9}
\end{gathered}
$$




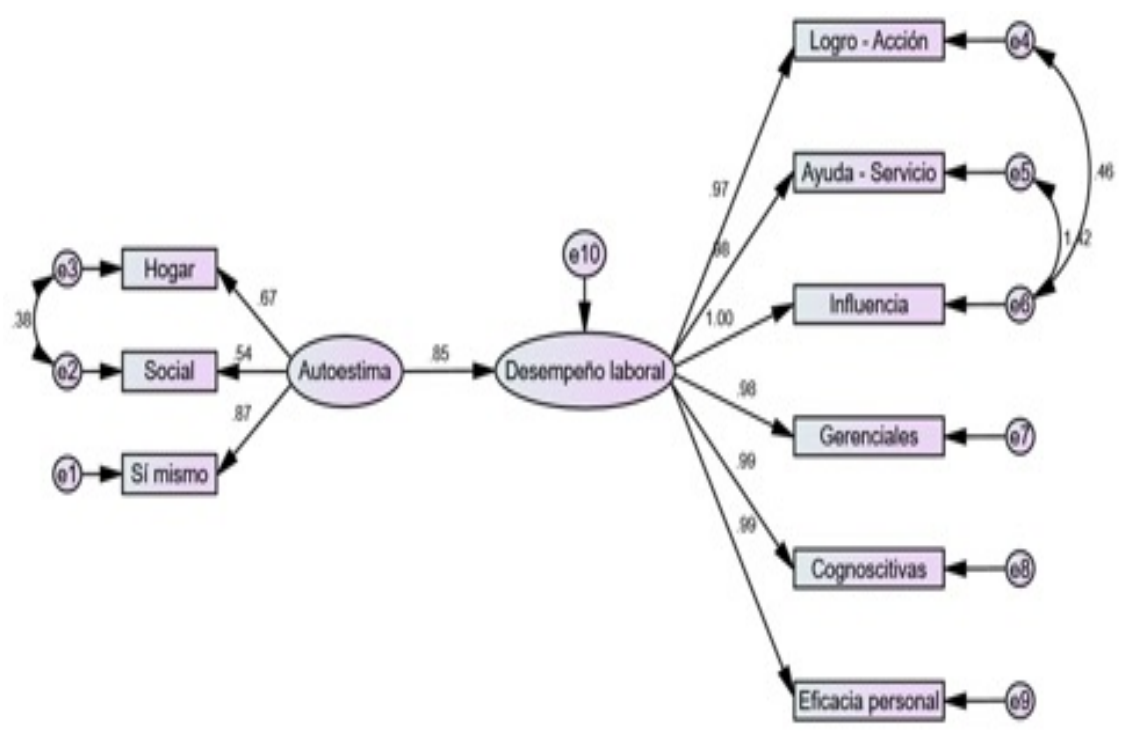

Figura 1. Modelo de ecuaciones estructurales de la autoestima y el desempeño laboral de los trabajadores de las áreas de Ciencias Básicas e Ingeniería, 2020

\section{Tabla 3}

Modelo de medida de la autoestima de los trabajadores de las áreas de Ciencias Básicas e Ingeniería, 2020

\begin{tabular}{cccccccc}
\hline & \multirow{2}{*}{ Relación } & & \multicolumn{2}{c}{ Coeficiente de Regresión } & \multirow{2}{*}{ E.S. } & C.R. & P \\
& & & Estimado & Estándar & & & \\
\hline Sí mismo & $\leftarrow$ & Autoestima & 1.000 & 0.631 & & & \\
Social & $\leftarrow$ & Autoestima & 0.262 & 0.646 & 0.034 & 7.691 & $* * *$ \\
Hogar & $\leftarrow$ & Autoestima & 0.542 & 0.892 & 0.073 & 7.454 & $* * *$ \\
\hline
\end{tabular}

E.S. (error estándar); C.R. (Coeficiente de regresión)

En la Tabla 3, con un nivel de significancia de $p=0.05$, se observa que la autoestima se relaciona de manera positiva con la dimensión sí mismo (0.631), de manera positiva con la dimensión social (0.646) y de manera positiva con la dimensión hogar (0.892) de la autoestima.
Por otro lado, se observa que el predominante es la dimensión hogar por presentar mayor coeficiente estandarizado, siendo esta dimensión la que mayor resalta en los trabajadores de las áreas de Ciencias Básicas e Ingeniería. 
Tabla 4

Indicadores de bondad de ajuste del modelo de medida de la autoestima de los trabajadores de las áreas de Ciencias Básicas e Ingeniería, 2020

\begin{tabular}{cccc}
\hline Nombre & Medida de ajuste & Valor & Límite aceptable \\
\hline $\begin{array}{c}\text { Índice de ajuste normado } \\
\text { Índice de ajuste } \\
\text { comparativo }\end{array}$ & NFI & 1,000 & $\geq 0,90$ \\
$\begin{array}{c}\text { Índice ajustado de } \\
\text { bondad de ajuste }\end{array}$ & AGFI & 1,000 & $\geq 0,90$ \\
$\begin{array}{c}\text { Índice relativo de ajuste } \\
\text { Raíz cuadrada del error } \\
\text { cuadrático medio }\end{array}$ & RFI & 1,000 & $\geq 0,85$ \\
\hline
\end{tabular}

En la Tabla 4, se observa que el modelo de medida es aceptable debido a que cumple con todos los indicadores de bondad de ajuste, lo cual indica que se tendría un ajuste perfecto; es decir, que dicho modelo no contaría con errores de estimación; por lo tanto, se debe tener cuidado en la interpretación a nivel más profundo.

\section{Modelo de medida}

Si mismo $=0.63 \times$ Autoestima $\mathrm{e}_{1}$ Social $=0.65 \times$ Autoestima $+e_{2}$ Hogar $=0.89 \times$ Autoestima $+\mathrm{e}_{3}$

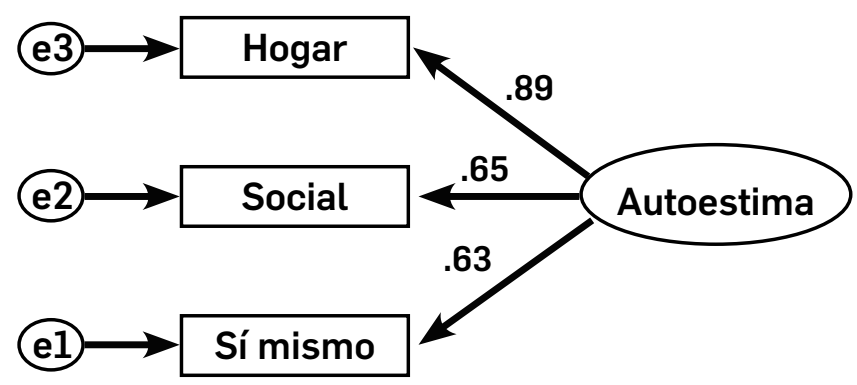

Figura 2. Modelo de medida de la autoestima de los trabajadores de las áreas de Ciencias Básicas e Ingeniería, 2020 


\section{Tabla 5}

Modelo de medida del desempeño laboral de los trabajadores de las áreas de Ciencias Básicas e Ingeniería, 2020

\begin{tabular}{|c|c|c|c|c|c|c|c|}
\hline \multirow{2}{*}{\multicolumn{3}{|c|}{ Relación }} & \multicolumn{2}{|c|}{ Coeficiente de Regresión } & \multirow{3}{*}{$\begin{array}{l}\text { E.S. } \\
0.032\end{array}$} & \multirow{3}{*}{$\begin{array}{l}\text { C.R. } \\
64.541\end{array}$} & \multirow{3}{*}{$\begin{array}{c}\mathrm{P} \\
* * *\end{array}$} \\
\hline & & & \multirow{2}{*}{$\begin{array}{c}\text { Estimado } \\
2.065\end{array}$} & \multirow{2}{*}{$\begin{array}{c}\text { Estándar } \\
0.986\end{array}$} & & & \\
\hline Ayuda-servicio & $\leftarrow$ & Desempeño laboral & & & & & \\
\hline Influencia & $\leftarrow$ & Desempeño laboral & 1.375 & 1.031 & 0.026 & 52.456 & $* * *$ \\
\hline Gerenciales & $\leftarrow$ & Desempeño laboral & 1.081 & 0.973 & 0.023 & 47.102 & $* * *$ \\
\hline Cognoscitivas & $\leftarrow$ & Desempeño laboral & 1.026 & 0.972 & 0.023 & 45.267 & $* * *$ \\
\hline Logro-acción & $\leftarrow$ & Desempeño laboral & 1.000 & 0.971 & & & \\
\hline Eficacia personal & $\leftarrow$ & Desempeño laboral & 1.063 & 0.971 & 0.024 & 43.668 & $* * *$ \\
\hline
\end{tabular}

E.S. (error estándar); C.R. (Coeficiente de regresión)

En la Tabla 4, se observa que el modelo de medida es aceptable debido a que cumple con todos los indicadores de bondad de ajuste, lo cual indica que se tendría un ajuste perfecto; es decir, que dicho modelo no contaría con errores de estimación; por lo tanto, se debe tener cuidado en la interpretación a nivel más profundo.

\section{Modelo de medida}

LogroAcción= 0.97xDesempeñoLaboral $+e_{4}$ AyudaServicio $=0.99 \times$ DesempeñoLaboral $+e_{5}$ Influencia $=1.03 \times$ DesempeñoLaboral $+e_{6}$ Gerenciales $=0.97 \times$ DesempeñoLaboral $+e_{7}$ Cognitivas $=0.97 \times$ DesempeñoLaboral $+e_{8}$ EficaciaPersonal $=0.97 \times$ DesempeñoLaboral $+e_{9}$

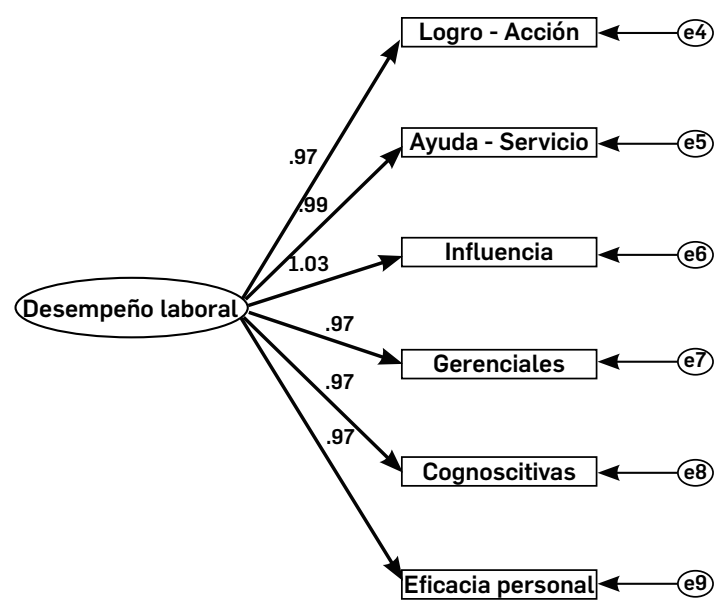

Figura 3. Modelo de medida del desempeño laboral de los trabajadores de las áreas de Ciencias Básicas e Ingeniería, 2020 


\section{DISCUSIÓN}

Los trabajadores administrativos de la Universidad Nacional Mayor de San Marcos debido al trabajo extenuante y exigido han expresado su cansancio y su desconfianza en el sistema laboral de la universidad. Es por ello, que dicha latente situación de trabajo estaría afectando el uso de sus conocimientos, intuiciones y percepciones como herramientas para enfrentar con sosiego y serenidad las situaciones y conflictos cotidianos. Ello ha afectado el adaptarse y aceptar los nuevos retos y no sentirse intimidado ante situaciones específicas; por lo tanto, la baja autoestima no ayuda a los trabajadores a que sean capaces de transmitir a los demás colaboradores los mensajes de forma clara y en el momento adecuado lo que piensan y sienten, todo ello es refrendado a partir de los resultados hallados donde el autoestima presenta un efecto directo en el desempeño laboral de estos trabajadores ya que servidores con niveles altos de autoestima tiene un alto desempeño laboral (Pastor \& Alvan, 2017; Marlon, 2018), y trabajadores con bajo autoestima se corresponde principalmente con el mal desempeño laboral (Huamán, 2020). mermando de esta manera las capacidades y potencialidades que cada uno de los administrativos tiene para desempeñar sus funciones, y de esa manera aprovechar al máximo en favor de una buena gestión institucional como resultado de un buen desempeño laboral, ello exige confianza por parte de los funcionarios y libertad parael desarrollo de todas las actividades enmarcado dentro del marco normativo de sus funciones. Estos resultados son semejantes a los encontrados por Romero (2018) y Pacheco (2017) y discordantes con Cáceres (2017), en la que encontró que no había relación significativa entre la autoestima y desempeño laboral de los trabajadores, ya que dichos trabajadores presentan conceptos débiles de sí mismos sin ejercer control sobre sus emociones, sin poder autoevaluar su desempeño evidenciando su inseguridad.

Así mismo, el modelo adecuaciones estructurales aporta información multivariada de las relaciones estructurales del constructo de la autoestima y del constructo del desempeño laboral, todos ellos avalados por medidas de bondades de ajuste aceptables mostrando de esta manera que el modelo estimado es adecuado para su interpretación descriptiva de la realidad estudiada, siendo semejantes a los resultados encontrados por Samperio (2019), Manzano (2017), y Medrano (2017).

\section{CONCLUSIONES}

La autoestima influye de manera positiva en el desempeño laboral de los trabajadores del área de Ciencias e Ingeniería de la Universidad Nacional Mayor de San Marcos.

Las dimensiones del sí mismo se relacionan con la autoestima de manera directa mostrando su unicidad como constructo.

Las dimensiones del desempeño laboral se relacionan de manera positiva con el desempeño laboral mostrando de esta manera la unicidad del constructor.

\section{AGRADECIMIENTO}

Un agradecimiento especial a escuela de Posgrado de la Universidad Nacional del Santa y mi familia por su apoyo incondicional.

\section{REFERENCIAS BIBLIOGRÁFICAS}

Aguilar, E. (2018). Familias con autoestima. Árbol

Alcántara, J. (2009). Educar la autoestima. CEAC.

Amperio, K. (2019). Ecuaciones estructurales en los modelos educativos: características y fases en su construcción. Universidad de Guadalajara. http:// dx.doi.org/10.32870/Ap.v11n1.1402.

Byrme, B. (2010). Structural Equation Modeling with AMOS. Routledge Taylor \& Francis Group.

Blanco, A. (2007). Trabajadores competentes. Introducción y reflexiones sobre la gestión de recursos humanos por competencias. ESIC.

Bentler, PM y Bonett, DG (1980). Pruebas de significancia y bondad de ajuste en el análisis de estructuras de covarianza. 
Psychological Bulletin, 88 (3), 588-606. DOI:https://doi.org/10.1037/00332909.88.3.588

Cáceres, R. (2017). Autoestima y desempeño laboral docente en la institución educativa secundaria Nuestra Señora De Alta Gracia, Ayaviri2016 [Tesis demaestría],Universidad Nacional del Altiplano. Disponible en: http://repositorio.unap.edu.pe/bitstream/ handle/UNAP/6228/EPG891-0089101. pdf? sequence $=1 \&$ is Allowed $=y$

Cequea, J. \& Rodriguez, D. (2012). Modelo de ecuaciones estructurales. Manzano.

Comisión Económica para América Latina y el Caribe (2016). Indicadores laborales de América Latina y el Caribe continúan deteriorándose en medio de contracción económica regional. https://www. eleconomistaamerica.pe/sociedadeAm-peru/noticias/7902680/10/16/ Indicadores-laborales-de-America-LatinaelCaribe-continuan-deteriorandose-enmedio-de-contraccion-economica-regional. html.pdf

Druker, P. (2002). Los desafíos de la gerencia para el siglo XXI. Norma.

Liderman (2018). Autoestima en el trabajo: la clave del éxito laboral. https://www. liderman.com.pe/blog-autoestima-trabajoexito-laboral/pdf.

Manzano, P. (2017). Introducción a los modelos de ecuaciones estructurales. Investigación en Educación Médica. https://doi. org/10.1016/j.riem.2017.11.002.

Medrano, L. (2017). Aproximación conceptual y práctica a los Modelos de Ecuaciones Estructurales. Disponible en: http://www. scielo.org.pe/pdf/ridu/v11n1/a15v11n1.pdf

Organización Internacional del Trabajo. (2017). Desafíos de la productividad laboral. $\quad$ https://www.ilo.org/wcmsp5/ groups/public/---americas/---ro-lima/--sro-santiago/documents/publication/ wcms_549576.pdf.

Organización Internacional del Trabajo. (2016). Panorama Laboral 2016. https:// www.ilo.org/americas/publicaciones/ panoramalaboral/WCMS_537803/lang-es/index.html

Ortiz, M y Montserrat, F. (2017). Modelo de Ecuaciones Estructurales: Una guía para ciencias médicas y ciencias de la salud. Sociedad Chilena de Psicología Clínica. https://teps.cl/index.php/teps/article/ view/230

Pacheco, H. (2017). Modelo de clima organizacional y su relación con el desempeño laboral en las PYMES hoteleras de Riobamba. [Tesis doctoral], Universidad Nacional Mayor de San Marcos.

Panesso, K \& Arango, M. (2017). La autoestima, proceso humano. https://aprendeenlinea. udea.edu.co>Psyconex> article>download. pdf.

Pernía, K \& Carrera, M. (2014). Correlación entre las competencias y el desempeño laboral. EAE.

Preciado, A. (2006). Modelo de Evaluación Por Competencias Laborales. Universidad Panamericana.

Ramírez, V. (2008). Autoestima. Paulinas.

Rodríguez, M. (2015). La autoestima: clave del éxito personal. Manual Moderno. 